\title{
Selecting patterns and features for between- and within- crop-row weed mapping using UAV-imagery
}

\author{
María Pérez-Ortiz ${ }^{\mathrm{a}, \mathrm{c}, *}$, José Manuel Peña ${ }^{\mathrm{a}}$, Pedro Antonio Gutiérrez ${ }^{\mathrm{b}}$, Jorge \\ Torres-Sánchez ${ }^{\mathrm{a}}$, César Hervás-Martínez ${ }^{\mathrm{b}}$, Francisca López-Granados ${ }^{\mathrm{a}}$ \\ ${ }^{a}$ Institute for Sustainable Agriculture, CSIC, P.O. Box 4084, 14080-Córdoba, Spain \\ ${ }^{b}$ Department of Computer Science and Numerical Analysis, University of Córdoba, \\ Rabanales Campus, C2 building, 14071-Córdoba, Spain \\ ${ }^{c}$ Department of Mathematics and Engineering, Universidad Loyola Andalucía, third \\ building, 14004-Córdoba, Spain
}

\begin{abstract}
This paper approaches the problem of weed mapping for precision agriculture, using imagery provided by Unmanned Aerial Vehicles (UAVs) from sunflower and maize crops. Precision agriculture referred to weed control is mainly based on the design of early post-emergence site-specific control treatments according to weed coverage, where one of the most important challenges is the spectral similarity of crop and weed pixels in early growth stages. Our work tackles this problem in the context of object-based image analysis (OBIA) by means of supervised machine learning methods combined with pattern and feature selection techniques, devising a strategy for alleviating the user intervention in the system while not compromising the accuracy. This work firstly proposes a method for choosing a set of training patterns via clustering techniques so as to consider a representative set of the whole field data spectrum for the classification method. Furthermore, a feature selection method is used to obtain the best discriminating features from a set of several statistics and measures of different nature. Results from this research show that the proposed method for pattern selection is suitable and leads to the construction of robust sets of data. The exploitation of different statistical, spatial and texture metrics represents a new avenue with huge potential for between and within crop-row weed mapping via UAV-imagery and shows good synergy when complemented with OBIA. Finally, there are some measures (specially those linked to vegetation indexes) that are of great influence for weed mapping in both sunflower and maize crops.
\end{abstract}

Keywords: Remote sensing, unmanned aerial vehicles (UAV), weed detection, object based image analysis

\footnotetext{
${ }^{*}$ Corresponding author at: Institute for Sustainable Agriculture, CSIC, P.O. Box 4084, 14080-Córdoba
} 


\section{Introduction}

Sunflower is nowadays the third most important herbaceous crop in Spain when considering cultivated area. Demand of its seeds and the high consumption of sunflower oil have consolidated the importance of this crop in the agriculture

5 of Spain and other European countries. Maize also plays an important role in the Spanish fields (and worldwide, e.g. in the Mediterranean basin, ArgentinaBrazil and the corn-belt in the USA, among others). The relevance of this crop worldwide comes from its wide uses.

Although the impact that these crops have in the Spanish agricultural sector is clear, weeds are usually responsible for a large reduction in potential crop yields (approximately 35\%). Because of this, nowadays, most farmers in the EU rely on synthetic herbicides, usually providing a weed control efficacy of $75 \%$ (Oerke, 2006). The percentage of herbicides used in maize and sunflower with respect to the total of pesticides is large ( $86.5 \%$ for maize and $87.3 \%$ for sunflower),

15 the optimised use of these herbicides being vital for the agricultural scenario. Although weeds are distributed in patches and there are very clear economical and environmental risks from the over-application of these herbicides, the most common procedure is to apply them to the whole field. The cost of these herbicides usually accounts for $40 \%$ of the cost of all of the chemicals applied to

20 agricultural land in Europe (European Crop Protection Association (ECPA), 2015). This economic factor together with environmental concerns have led to the creation of the European legislation on the Sustainable Use of Pesticides (Regulation (EC) 1107/2009 and Directive 2009128/EC, 2009; Horizon 2020). The inclusion of these guidelines has been parallel to the introduction of patch

25 spraying in the machinery, which has enabled the feasibility of site-specific weed management (SSWM) based on weed coverage maps obtained via ground sampling, proximal sensing or remote sensing. In this sense, the key component of SSWM is to provide precise and timely weed maps for an appropriate early weed control.

30 In early growth stages, the spectral and appearance characteristics of both crops and their infesting weeds are similar, thus imposing additional difficulties for their differentiation. The mapping of weeds has been addressed by most previous works by detecting weeds at late growth stage (e.g. flowering) using piloted aircrafts or QuickBird satellite imagery (Castillejo-González et al., 2014.

35 Gutiérrez et al., 2008), although the spatial resolution of these platforms is not suitable for seedling detection (pixel size around $50 \mathrm{~cm}$ and $2.6 \mathrm{~m}$ for piloted aircrafts and QuickBird satellite, respectively). Nonetheless, a new aerial platform has recently joined the traditional ones, known as the Unmanned Aerial Vehicle (UAV) Moranduzzo \& Melgani, 2014). Different studies have highlighted the 40 advantages of UAVs over airborne or satellite equipment (Lucieer et al., 2014, Peña et al., 2013), specially a minor cost, a higher flexibility in flight scheduling

E-mail addresses: i82perom@uco.es, jmpena@ias.csic.es, pagutierrez@uco.es, jtorres@ias.csic.es, chervas@uco.es, flgranados@ias.csic.es 
and a better spatial resolution. These advantages make UAVs a very useful tool to perform field studies for crop and weed monitoring at early crop and weed phenological stage (Torres-Sánchez et al. 2013), which is a classic limitation of the traditional remote sensing platforms.

Previous methods in the literature have been designed with the purpose of remote weed mapping using manually-defined rules and have shown great promise in detecting weeds between-crop-rows (Peña et al. , 2013; Pérez-Ruiz et al. 2015), but the identification of weeds within-crop-rows still remains an

${ }_{50}$ open challenge. The reason is that the algorithm designed for weed detection must be highly robust because very similar seedling crop and weed plants emerge mixed within the crop row. In this sense, this paper studies the use of supervised machine learning methods for constructing a model for weed identification. This is one of the main novelties of this paper, because machine learning meth5 ods have been mostly used with the purpose of remote vegetation mapping in on-ground studies (Tellaeche et al. 2011, Burgos-Artizzu et al., 2011) or using piloted platforms but have uniquely been used with UAV-imagery in two preliminary works which showed the great potential of these techniques Hung et al. 2014, Pérez-Ortiz et al. 2015b). Our present work considers these previ-

${ }_{60}$ ous results and tries to deal with some of the problems that have been identified when using object-based image analysis (Blaschke, 2010) (OBIA), a strategy that have shown better performance than the pixel-based approach in preliminary results (Pérez-Ortiz et al., 2015b). Roughly speaking, OBIA is devoted to the division of remote sensing imagery into meaningful sets of pixels (known 65 as objects) which are considered as similar based on a measure of homogeneity Blaschke, 2010).

In a preliminary conference work (Pérez-Ortiz et al. 2015a), a first hypothesis that we studied was whether OBIA provided more robust results and how many objects should be labelled. The results showed that OBIA was beneficial, 70 both in terms of performance and computation time. This result was obtained only labelling 100 objects per class (randomly selecting objects and manually labelling them using three classes: soil, weeds and crop). The problem of this approach was that the size of the field image in this type of applications is usually large (and so is the number of objects produced), being difficult the se-

75 lection of a representative set of patterns of the whole experimental field. In this way, our classification method encountered problems when classifying objects not contemplated in the described spectral range (e.g. rocks) (Pérez-Ortiz et al. 2015a) and demanded a lot of effort from the user to select a suitable training set of patterns. The results also showed that there were other features, apart 80 from the mean and standard deviation of the object, that had a great impact in the classification and that the use of machine learning helped distinguishing weeds within-crop-rows and creating more robust learning models. Moreover, differences in performance occurred when factors such as flight height, sensor and classification method were considered. The results showed that the opti-

85 mal flight height was $30 \mathrm{~m}$ using preferably a visible sensor and an advanced classification method.

In this paper, we extend the previously mentioned work by trying to answer 
additional questions. Firstly, whether it is possible to develop an automatic method to select the most representative objects of the field, including the whole 90 object spectrum and providing preliminary labels. Ideally, this method would perform better than the random choice of objects. Secondly, since objects are classification units that entail further information than single pixels, we study the best method for characterising them. We consider the use of histograms, as an alternative to the common approach of considering the mean and standard 95 deviation. Finally, this paper also considers a wide range of features (statistical, texture-based, geometrical and spatial) and analyses the best suited ones. The problem of feature selection in high spatial resolution imagery has been researched for urban-related environments (Herold et al. 2003), but there has not been much work in vegetation mapping (Yu et al., 2006) (and more specifically, weed mapping), mainly because of the proximity of the spectral signatures for different species and the difficulties in capturing texture features in vegetation (Carleer \& Wolff, 2004). We also try to validate the hypothesis that the same data features are useful for weed mapping considering different crops (sunflower and maize, which are broadleaved-dicotiledoneous and grass-monocotiledoneous 105 crops, respectively). Note that the base problem of this paper could be addressed using binary classification, however, crop detection is also an important challenge for a wide range of applications (such as plant counting, sowing failures detection or patch spraying positioning). Therefore, this problem is considered as a three-class task (weeds, crops and non-vegetation).

In this way, the experiments of this paper have three main purposes:

- To test whether the proposed method for object selection is useful to construct a reliable training set and to analyse the number of patterns needed to train the model.

- To test the use of histograms as opposed to the idea of using statistical metrics to simplify the objects.

- To ascertain which of the features considered are more useful for weed mapping in sunflower and maize crops via remote sensing imagery. To do so, a wide set of 40 features is selected and a procedure of feature selection is considered.

${ }_{120}$ To conduct this study, different datasets have been created from sunflower and maize fields naturally infested by weeds and the results are validated by the use of the well-known SVM classifier.

The paper is organised as follows: section 2 shows a description of the data acquisition process; section 3 exposes the weed mapping system proposed in this work; section 4 describes the experimental study, and Section 5 analyses the results obtained; Section 6 provides a discussion of the main findings; and finally, section 7 outlines the conclusions and future work. 


\section{Data acquisition and processing}

This section outlines the information concerning the data acquisition and

130

\author{
UAV and the sensor considered, while the second one is focused on the image
}

image processing steps. The first subsection explains the characteristics of the mosaicking process.

The UAV system was tested in a sunflower field situated at the private farm La Monclova (South of Spain), and in a maize field situated at the private farm when post-emergence herbicide or other control techniques are recommended in these crops. The crops were at the stage of $4-6$ leaves unfolded. The fields were naturally infested by weeds, and they had a similar size or, in some cases, the weeds were even smaller than the crop plants. More specifically, the sunflower 140 field was infested by pigweed (Amaranthus blitoides), mustard (Sinapsis arvensis) and bindweed (Convolvulus arvensis), whereas the maize field was infested by saltwort (Salsola kali). An experimental plot of $100 \times 100 \mathrm{~m}$ was delimited within the crop-fields to perform the flights. The coordinates of each corner of the flight area were collected using a global position system (GPS) to prepare

\section{1. $U A V$ and sensor specifications}

A quadrocopter platform with vertical take-off and landing, model md4-1000 (microdrones GmbH, Siegen, Germany), was used to collect the set of aerial images over the above-mentioned crop-fields. The flight route was programmed and controlled the UAV software to allow the UAV reach the required altitude and degree of image overlapping for mosaicking. The imagery was collected at an altitude of 30 meters, resulting in an image spatial resolution of $1.4 \mathrm{~cm}$. A low cost still camera, model Olympus PEN E-PM1 (Olympus Corporation, Tokyo, Japan), was used. At the moment of each shoot, the on-board computer system 155 records a timestamp, the GPS location, the flight altitude, and vehicle principal axes (pitch, roll and heading). The Olympus camera acquires 12-megapixel images in true colour (R, G and B bands) with 8-bit radiometric resolution and is equipped with a 14-42 $\mathrm{mm}$ zoom lens (in our case we used a focal length of $14 \mathrm{~mm}$ ). A sequence of $60 \%$ end or longitudinal lap and $30 \%$ side or lateral lap imagery was collected to cover the whole experimental field corresponding to each crop (Figure 1). Detailed information about the configuration of the UAV flights and specifications of the vehicle and sensors can be found in (TorresSánchez et al. 2013).

\subsection{Image mosaicking}

165 Overlapped images were collected for this study to cover the whole studied field. This is because UAVs fly at low altitudes and therefore can not cover the whole field in one image, this causing the need to take a sequence of multiple overlapping (end-lap or lateral-lap and side-lap or longitudinal-lap) images. A necessary step is the combination of these individual images via a process of 170 


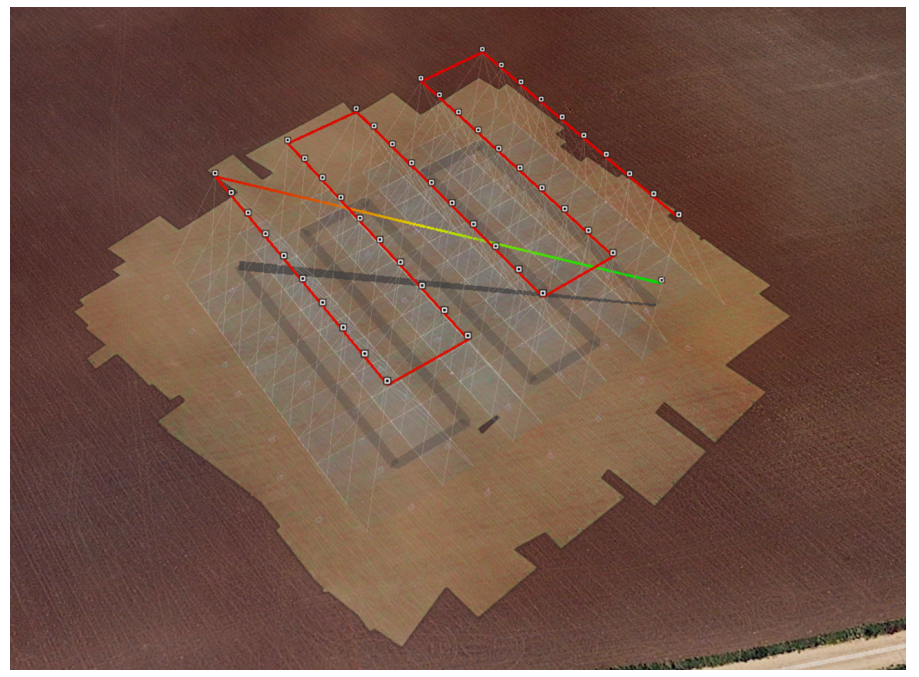

Figure 1: Flight plan and mosaicking results for the sunflower field at 30m of flight altitude. Red line represents the flight route, and the square points indicate the position of camera shots. Grey transparent rectangles simulate the projection over the terrain of each aerial image, and less transparent areas indicate the image overlap.

Edition (Agisoft LLC, St. Petersburg, Russia) software was employed for this task.

1. Image acquiquisition

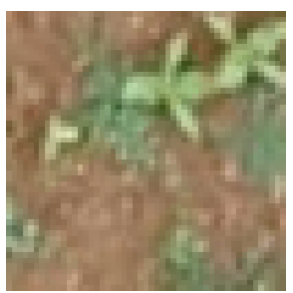

2. Image segmentation

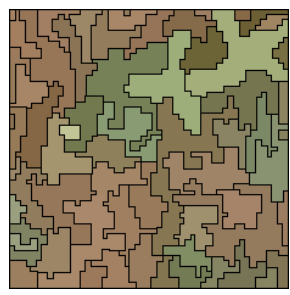

3. Data labelling

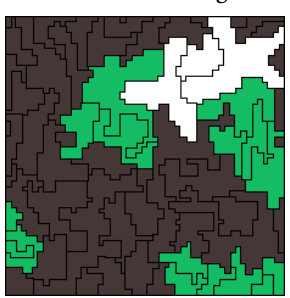

Figure 2: Representation of the different steps in sunflower for the procedure considered preceding the classification: image acquisition, segmentation of the image and data labelling.

\section{Classification strategy}

This section comprises the set of steps considered for the purpose of weed mapping. The steps are summarised in Figure 2. The first step corresponds to the segmentation of the mosaicked image in a set of objects. From these objects, a subsample is labelled using the unsupervised pattern selection method proposed and the automatic labelling is then supervised by an expert. The next step is the computation of different characteristics for the classification of the objects. In this case we consider two approaches: the characterisation of the 
objects using colour histograms and the use of several measures previously used in the remote-sensing literature (in addition to other two proposed metrics). Finally, the last step of this system is the classification of the data via machine learning techniques and the application of a feature selection approach.

\subsection{Object-based image analysis}

Object-based image analysis (OBIA) is a sub-discipline of the research field of image analysis. OBIA is mainly devoted to divide or segment imagery into meaningful objects by assessing their characteristics. In this sense, it is related to other concepts of image analysis, such as image segmentation (Ghamisi et al. 2012) or super-pixels. However, being mainly focused on remote sensing applications, OBIA considers homogeneity measures that differ based on the application considered. Objects are image regions derived by one or more criteria of homogeneity in one or more dimensions (i.e., characteristics of the feature space). In high spatial resolution imagery, a group of pixels can represent the characteristics of the field considered better than single pixels $(\mathrm{Yu}$ et al. 2006). In this paper, we make use of this technology, treating each of the objects as a minimum classification unit. Although segmentation techniques are not new in the area of computer vision, they have been extensively applied to classify remote sensing data quite recently, given also the relative novelty of 200 remote sensing platforms that provide high-spatial resolution images. Several advantages of objects over single pixels have been found (Blaschke, 2010): objects can entail further information apart from the spectral characteristics, the use of objects as basic units reduces the computational load of the classification method (Pérez-Ortiz et al., 2015a) and enables the user to consider more complex techniques, and finally, image objects help to overcome the common "salt and pepper effect".

The segmentation algorithm used is the one implemented in Bunting et al. 2014), given that it is focused on the remote sensing setting, designed for largescale data and included in an open source project. This method is based on the generation of spectrally similar units with a minimum object size. The procedure uses the well-known $k$-means clustering method (Jain, 2010) and an objectrefining step (to avoid very small objects). The algorithm presents two key parameters: the number of clusters $k$ and the minimum object size. The scale of the segmentation is controlled by $k$, where smaller values produce larger objects. These parameters have been optimised using the Johnson \& Xie (2011) method for measuring segmentation quality, which, in conjunction with the segmentation algorithm used, has been seen to lead to promising results, reaching the ones of other methods used in other proprietary software (for example, eCognition) (Clewley et al. 2014).

The first two images in Figure 2 show a selection of the sunflower field and the result of the segmentation algorithm in this subimage, respectively.

\subsection{Pattern selection and data labelling method proposed}

Supervised classification methods require a labelled set of data to train the learning model. However, how to choose automatically which patterns to label 
225 is an important task. In this paper, we propose a new method that selects the patterns that the user has to label. We consider an intermediate number of training patterns (100 patterns per class, as we saw that this can lead to good results, Pérez-Ortiz et al. (2015a)). The reason is that the labelling task can be very tedious for the user, and one of the purposes of this paper is to

230 simplify it. The proposed methodology makes use of the whole set of objects and selects the most representative patterns based on a well-known vegetation index, a thresholding algorithm and a data clustering technique.

The vegetation index used is the Excess Green (ExG) Woebbecke et al. 1995), which can be used to discriminate if a pixel corresponds to vegetation or

235 soil. This index can be computed as follows:

$$
\mathbf{E}=2 \mathbf{G}^{*}-\mathbf{R}^{*}-\mathbf{B}^{*}
$$

where $\mathbf{R}^{*}=\frac{\mathbf{R}}{\mathbf{R}+\mathbf{G}+\mathbf{B}}, \mathbf{G}^{*}=\frac{\mathbf{G}}{\mathbf{R}+\mathbf{G}+\mathbf{B}}, \mathbf{B}^{*}=\frac{\mathbf{B}}{\mathbf{R}+\mathbf{G}+\mathbf{B}}, \mathbf{E}$ is the ExG output matrix, and $\mathbf{G}, \mathbf{R}$ and $\mathbf{B}$ are the matrices associated to the green, red and blue spectral channels, respectively. We use this index as an initial estimator of the class of each object (weeds, crop and non-vegetation). Firstly, we divide the 240 pixels in two levels (vegetation and non-vegetation) using the Otsu's thresholding algorithm (Otsu, 1979). Then, for the vegetation pixels, we divide the data in other two levels (in this case, one level refers to weeds and the other to crops, assuming that they are represented by differences in the vegetation index). With these steps, we would have all pixels prelabelled.

245 Once the pixels are classified, each object is assigned to one of the classes using different criteria (in our case using the mode). This process of initial labelling is essential for alleviating the user task, as only misclassified objects will have to be relabelled.

The next step is to perform a clustering analysis of each of the three pools of objects and select the centroid of each cluster in order to explore the whole data spectrum of each pool. This clustering is performed using the well-known $k$ means algorithm (Jain, 2010), where $k=100$ since we fixed the training number of patterns per class to 100. The characteristics used are the mean and standard deviation of the spectral data and the ExG vegetation index of each object (i.e., 255 eight data features in total). The centroids will be the selected objects to be labelled for each class.

Figure 3 shows the different steps of the proposed method for pattern selection. The main steps are the following: computation of the vegetation index (in our case, ExG); ExG thresholding via the Otsu's method; object labelling using the previous pixel classification based on the thresholds computed; and finally, clustering the data for each of the three groups followed by a refining step performed by the user.

\subsection{Object representation and classification method}

Objects are represented using two different approaches, which are analysed in the next two subsections. In both cases, Support Vector Machines (SVMs) will be used as the classification model, since it is one of the most successful 

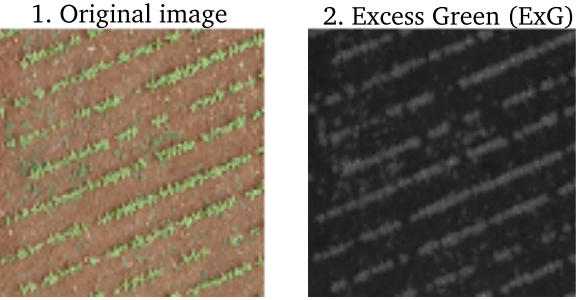

5. Data space for the weed class from the ${ }_{0.2}$ previous step (red, green and ExG channels).

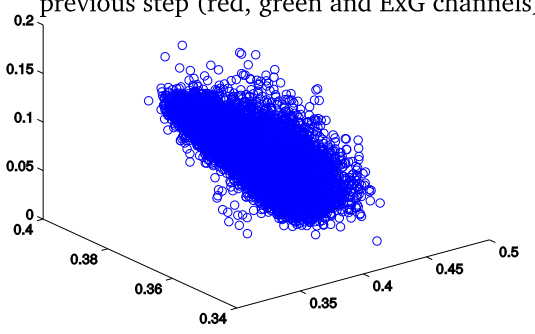

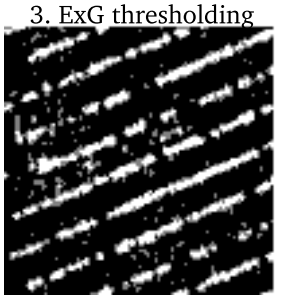
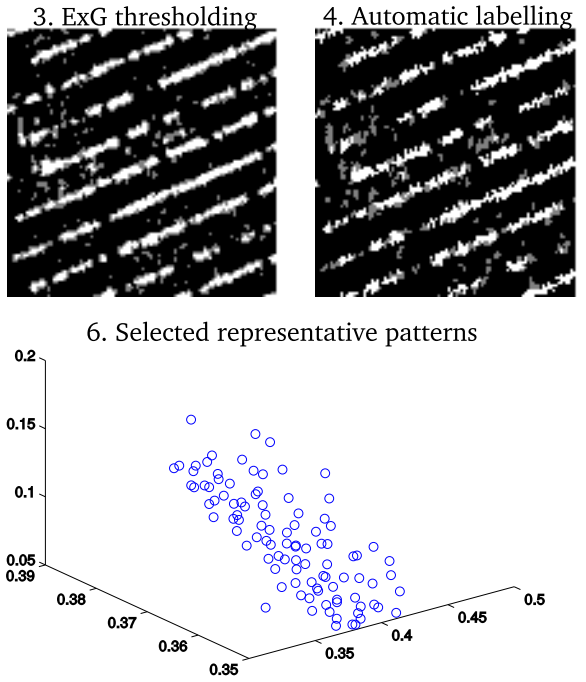

Figure 3: Representation of the different steps for the procedure considered for selecting the training patterns. Note that for step 5 a three-dimensional space has been used for representing the data, although the clustering is actually performed in an eight-dimensional data space. In steps 3 and 4, black coloured pixels represent non-vegetation, white ones represent crop, and grey ones correspond to weed.

machine learning methods and has been used for a wide range of applications concerning remote sensing (Mountrakis et al., 2011; Tellaeche et al., 2011).

\subsection{Representing objects by histograms}

The first approach is based on the use of colour histograms. Two procedures are tested:

1. The first approach is to use each bin of the histograms (255 bins for each channel) as an independent feature.

2. The second approach is to use a kernel function specifically designed for histograms. Kernel functions are used to devise nonlinear decision regions with originally linear methods such as SVMs. However, different kernels are suited for different purposes. In this paper histogram intersection kernel have been selected, which has been seen to be very helpful in the setting of histogram classification (Barla et al., 2003). We consider a different kernel for each histogram (each histogram is associated to one of the four channels), and we optimise a convex combination of these kernels by means of the kernel learning measure known as kernel-target alignment (Cortes et al. 2012). This is, we optimise a weight for each kernel such that the final kernel matrix $\mathbf{K}$ is computed as follows:

$$
\mathbf{K}=\lambda_{1} \cdot \mathbf{K}_{\mathrm{R}}+\lambda_{2} \cdot \mathbf{K}_{\mathrm{G}}+\lambda_{3} \cdot \mathbf{K}_{\mathrm{B}}+\lambda_{4} \cdot \mathbf{K}_{\mathrm{E}}, \quad \sum_{i=1}^{4} \lambda_{i}=1,
$$


where $\left\{\lambda_{1}, \lambda_{2}, \lambda_{3}, \lambda_{4}\right\}$ is the set of parameters to optimise, $\mathbf{K}_{\mathrm{R}}$ is the kernel matrix associated to the red channel, $\mathbf{K}_{\mathrm{G}}$ to the green channel, $\mathbf{K}_{\mathrm{B}}$ to the blue channel and $\mathbf{K}_{\mathrm{E}}$ to the ExG channel. This setting is useful for problems where each histogram comes from different sources of information and could have a different impact on the model.

\subsection{Representing objects by data features}

The second approach is based on the computation of a series of statistics and data features, which have been selected analysing other works ( $\mathrm{Yu}$ et al. 2006: Herold et al., 2003) and are computed for each object over the red, green, blue and ExG channels. A procedure of feature selection is then used to analyse the most suitable features and to validate the use of these in both crop fields.

\subsubsection{First order statistics}

Firstly, we consider several statistical features. The most common ones are the mean and standard deviation (we will refer to these as base statistics). We include these variables, as well as the kurtosis and skewness, which give us additional information about the data (peakedness and asymmetry, respectively). Sixteen statistical features are then considered (four statistical features for four different channels). We refer to this set as "All statistics" in the experiments.

\subsubsection{Second order statistics or textures}

Secondly, different textures were derived for each object considering each channel separately. To do so, we use the Gray-level Co-occurrence Matrix (GLCM) of each channel to describe image texture, as done for high resolution remote sensing (Yu et al. 2006, Herold et al. 2003). GLCM is an approximation of the joint probabilistic density function of pixel pairs and has been seen to be useful for describing textures of regions with irregular shapes.

Textures can be local or global and this choice depends on the characteristics to capture. Usually, a window size is considered and this size results in fine or coarse grained textures. The final texture value of the object is calculated as the mean texture value of its pixels. In our case, we consider object-specific window sizes. Rather than analysing the mean texture of the object, we consider the object as the texture unit, and the texture is calculated using the whole region in which the object is included, in order to include the information of the neighbouring pixels. Figure 4 shows an example of the window used to compute the texture metrics for a given object.

Four different texture descriptors have been selected according to the findings of the related bibliography (Yu et al. 2006: Herold et al., 2003). The considered descriptors are the following:

- Energy: The energy is a measure of local homogeneity. The higher the energy, the larger the homogeneity of the texture.

- Contrast: It measures the difference between the highest and lowest values of a contiguous set of pixels. 


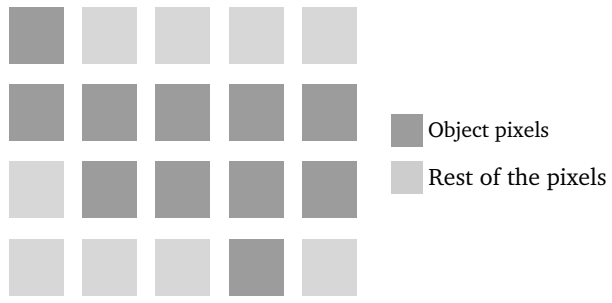

Figure 4: Representation of the window used for a given object (adapted to the object dimensionality) for the computation of textures.

- Correlation: It measures how correlated a pixel is to its neighbours over the whole image.

- Homogeneity: It is focused on the closeness of the distribution of elements in the GLCM to the GLCM diagonal.

Sixteen features are used (four texture features for four different channels).

\subsubsection{Geometric measures}

Thirdly, we also consider three features related to the object shape: number of pixels, maximum width in pixels and maximum height in pixels. We refer to these in the experiments as shape features.

\subsubsection{Spatial features}

One of the main hypothesis of this paper is that weed discrimination could be improved based on the relative location of weeds with respect to crop lines. Because of this, we also propose the use of two new sets of ExG-based spatial features which we have specifically designed for this problem:

- The first ExG-based features consider a method for crop row detection. More specifically, the mean and standard deviation of the distances of the pixels composing an object to the nearest crop row are used. Crop rows are computed using the Hough transform (HT), a widely used and powerful technique for detecting complex patterns of points in binary images. The HT method is applied to the ExG vegetation index using the thresholding computed in the data labelling step. From the results of the thresholding, non-vegetation pixels and weeds in the image are taken as 0 's, and crops are taken as 1's, in order to extract the crop rows via the HT method. Figure 5 shows a representation of the use of the HT for crop row detection in a subplot of both experimental fields. Images on the left part of the figure show the thresholded ExG and the ones on the right part the crop rows detected. The plots at the top correspond to sunflower and the ones at the bottom to maize. As can be seen, the use of the HT is satisfactory when using UAV-imagery even when there are sowing fails. Note that the HT has been widely used with on-ground studies (Tellaeche et al. 2011), but not much with remote sensing imagery, for which, up to our 
knowledge, it has only been used in two very recent studies Comba et al. 2015 Pérez-Ortiz et al. 2015b). For more information regarding how to use the Hough transform with UAV refer to Pérez-Ortiz et al. (2015b).
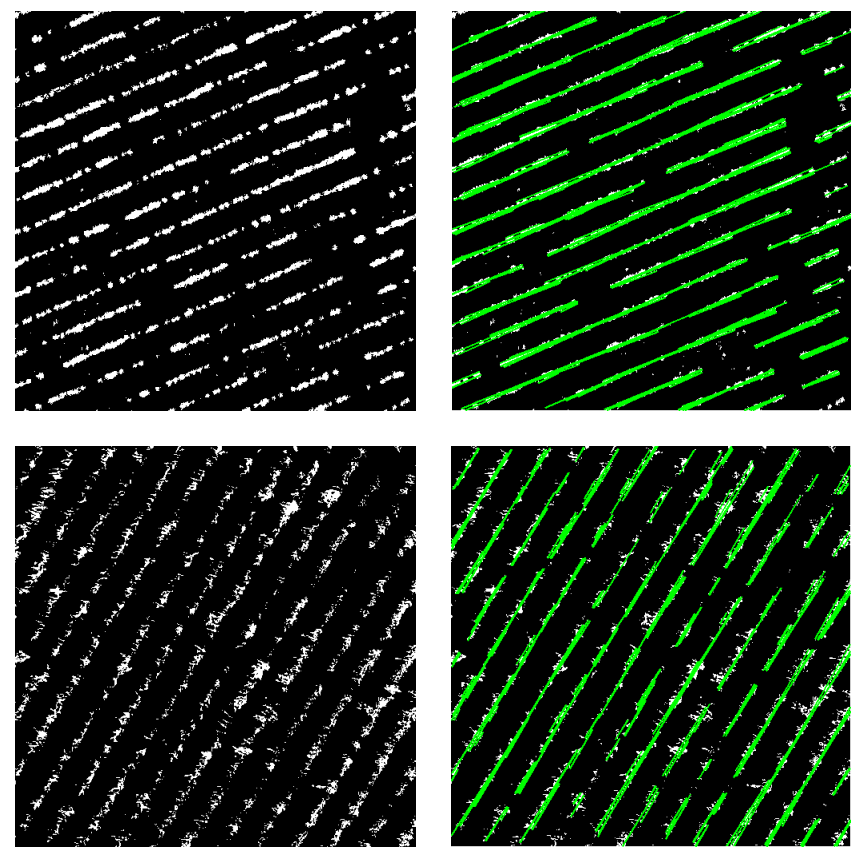

Figure 5: Representation of the use of the HT for crop row detection in sunflower (plots at the top) and maize crops (plots at the bottom). Images on the left part of the image show the thresholded ExG and the ones in the the right part the crop rows detected.

- The second set of features corresponds to the mean and standard deviation of the vegetation index of the pixels surrounding each object (using a buffer of 5 pixels). Because weeds are usually located in patches and crop pixels in crop rows, this feature could be of vital importance for weed detection. This feature was included to test whether the use of neighbour pixels could be of more interest for classifying the objects that the use of the crop row information previously presented, since the HT method demands considerable computation.

\subsubsection{Feature selection algorithm}

After computing the different features, the most discriminative ones are selected using a feature selection algorithm. The method considered (InfoGrainAttributeEval in Weka ( Hall et al. 2009) ) evaluates the worth of an attribute by measuring the information gain with respect to the class. We consider the first 10 attributes (which are the ones that present an information gain higher than $0.2)$ for both crops. 


\section{Experiments}

This section describes the experiment settings and the performance evaluation measures used.

\subsection{Parameter setting}

The two parameters of the segmentation method considered (number of clus-

(2011) method for measuring segmentation quality, both taking values within the following range: $\{30,50,100,200\}$.

The parameters associated to the Hough Transform have been fixed manually, since these do not influence the crop row detection to a great extent. The range of possible angle values for the lines has been fixed to $\left[10^{\circ}, 40^{\circ}\right]$ in the case of sunflower and to $\left[45^{\circ}, 85^{\circ}\right]$ for maize (the images should be analysed to fix this range). Another parameter, the number of lines to be detected, is set to 200 (as this number has been proven to work well for all images). In this paper, the minimum length of the lines and the gap between crop lines has been set to 50 .

The parameters associated to the Support Vector Machine (SVM) classifier are optimised using a stratified 5-fold procedure over the training set. The evaluation metric considered is the accuracy of the classification. The cost parameter is chosen from one of the following set: $\left\{10^{-3}, 10^{-2}, 10^{-1}, 10^{0}, 10^{1}, 10^{2}, 10^{3}\right\}$ (and so is the kernel parameter associated to SVM).

\subsection{Performance evaluation}

The training set has been constructed by 1) our proposal for object selection and 2) by random selection with the help of the user for labelling. Two experimental setups were considered to construct the training set, both consisting of 10 repetitions of a stratified holdout, but one with $75 \%$ of patterns for training and the other with $50 \%$. For these experiments, the test set was specifically designed to include 20 challenging objects of each class. From the 20 weed objects, 10 of them lie close to the crop row. From the 20 non-vegetation objects, we included 10 of them as rare objects (e.g. rocks). Note that the test set is $50 \%$ of training from the stratified holdouts is simply discarded).

In order to evaluate and compare the performance of the different methodologies, we use two different evaluation metrics:

- The well-known accuracy metric $(A c c)$ :

$$
A c c=\left(\frac{1}{N}\right) \sum_{n=1}^{N}\left(I\left(y_{n}^{*}=y_{n}\right)\right),
$$

where $I(\cdot)$ is the zero-one loss function, $y_{n}$ is the desired output for pattern $n, y_{n}^{*}$ is the prediction, and $N$ is the total number of patterns in the dataset. 
- Minimum Sensitivity $(M S)$ : This metric can be defined as the minimum value of the sensitivities for each class:

$$
M S=\min \left\{S_{i} ; i=0, \ldots, K\right\},
$$

where $S_{i}$ is the sensitivity for the $i$-th class. Sensitivity for class $i$ corresponds to the correct classification rate for this specific class.

\section{Results}

This section includes the information about the three different experiments considered: 1) validation of the method proposed for pattern selection; 2) use of 420 histograms for classification; 3) use of a set of different features for classification and a feature selection technique.

The procedure for obtaining the results is the following: 1) application of the selected segmentation technique to the image to obtain a set of objects; 2) selection of training and test objects (the training data is selected randomly or using our proposed clustering-based algorithm and the test data is selected manually); 3) computation of data features (complete histograms or different sets of statistics); and 4) data classification via the SVM method. In the labelling process, we extracted two patterns per each cluster derived (the two patterns closest to the cluster centroid) in order to have more potential patterns available for the relabelling step. After applying the relabelling step (i.e. after the user verification of the labelled patterns), for the sunflower case, the number of relabelled or discarded patterns was 39 (3 initially labelled as non-vegetation, 27 labelled as weeds and 9 labelled as crop). For the maize case, the number of relabelled or discarded patterns was 74 (6 initially labelled as non-vegetation, 43553 labelled as weeds and 15 labelled as crop). The patterns were reorganised in such a way that each class was composed of 100 training patterns. From this result, it can be seen that our proposed method for prelabelling the patterns does not commit many errors, although a minimum user intervention is still necessary.

\subsection{Selection of training patterns in sunflower crop}

The experiments performed and the results obtained can be seen in Table 1 . Most experiments are performed using the sunflower field, and then validated with the maize case. The first experiment compares our proposed method for object selection with a random selection in sunflower. It can be seen that our approach leads to a better-defined and more robust training set (analysing the mean and standard deviation of both metrics considered). It can also be appreciated that the use of a lower number of patterns when randomly selecting them improves the results. This could mean that the information included is redundant or even not useful for the classification method. However, when 450 considering our approach, the overall performance deteriorates, which could mean that all training patterns are useful in this sense. Finally, only 50 patterns per class are enough to yield a sufficiently good performance. 
Table 1: Mean results obtained for the different approaches considered and the different experiments conducted. Two metrics are presented in this case: accuracy $(A c c)$ and minimum sensitivity $(M S)$.

\begin{tabular}{c|cc}
\hline \hline Dataset-Experiment & $A c c$ & $M S$ \\
\hline Experiment 1: Selection of training data in sunflower crop \\
\hline Random selection, 75\% of training data used & $76.17 \pm 5.83$ & $52.00 \pm 17.19$ \\
Proposed method, 75\% of training data used & $\mathbf{9 0 . 6 7} \pm \mathbf{2 . 3 8}$ & $75.50 \pm 8.64$ \\
Random selection, 50\% of training data used & $77.50 \pm 5.34$ & $58.00 \pm 11.35$ \\
Proposed method, 50\% of training data used & $89.67 \pm 4.07$ & $\mathbf{7 7 . 5 0} \pm \mathbf{9 . 2 0}$ \\
\hline Experiment 2: Use of histograms for classification in sunflower crop \\
\hline Base statistics & $\mathbf{9 0 . 6 7} \pm \mathbf{2 . 3 8}$ & $\mathbf{7 5 . 5 0} \pm \mathbf{8 . 6 4}$ \\
Base histograms & $61.67 \pm 2.61$ & $10.50 \pm 1.58$ \\
Proposed convex combination of histograms & $72.33 \pm 3.35$ & $56.50 \pm 5.80$ \\
\hline Experiment 3: Feature selection in sunflower \\
\hline Base statistics & $90.67 \pm 2.38$ & $75.50 \pm 8.64$ \\
All statistics & $92.33 \pm 2.25$ & $83.00 \pm 4.83$ \\
Statistics + Textures & $89.50 \pm 2.36$ & $79.50 \pm 5.99$ \\
Statistics + Textures + Shape & $84.50 \pm 2.84$ & $67.00 \pm 9.19$ \\
Textures + Shape + Spatial & $85.83 \pm 3.17$ & $70.00 \pm 7.82$ \\
Features selected & $\mathbf{9 5 . 5 0} \pm \mathbf{2 . 0 9}$ & $\mathbf{9 1 . 5 0} \pm \mathbf{3 . 3 7}$ \\
\hline Experiment 4: Feature selection in maize \\
\hline Base statistics & $77.00 \pm 1.89$ & $47.00 \pm 8.23$ \\
\hline & $76.67 \pm 1.36$ & $31.50 \pm 4.12$ \\
Features selected & $\mathbf{7 9 . 0 0} \pm \mathbf{1 . 9 6}$ & $\mathbf{5 2 . 5 0} \pm \mathbf{7 . 1 7}$ \\
\hline \hline
\end{tabular}

\subsection{Use of histograms for classification in sunflower crop}

The second experiment measures the difference between the use of the base statistics for characterising an object (i.e. mean and standard deviation) and the use of the whole set of histograms. Two approaches have been tested in this case, as previously stated. For the first case (the so-called "Base histograms"), each bin of the histograms is used as an independent feature. In the second option, we use a kernel specially designed for histogram classification and a method for selecting the optimum combination of these histograms. In this case, however, the conclusion is that further research should be devoted to design algorithms for histogram classification, because none of the two proposals is able to improve the results of the base statistics. Moreover, it can also be seen that it is important to treat histograms as such and classify these with specific methods rather than with standard methods.

\subsection{Feature selection in sunflower}

The third experiment shows the difference between the use of the abovementioned features. Firstly, the combination of the base statistics with the kurtosis and skewness metrics shows good synergy and reaches an outstanding 

ever, the inclusion of further information deteriorates the result, but this could be influenced by some non-meaningful features which include noise into the model. Considering the feature selection process, as one can see in Table 1, the set of features selected help to improve the results to a great extent (specially 475 when considering the $M S$ metric). The features selected by the information gain method can be seen in Table 2 (including the name of the feature and channel, ordered by the information gain obtained). Most of the selected features are associated to the vegetation index and the green channel. The mean of the blue channel is also important. Finally, shape features do not contribute useful ${ }_{480}$ information, as well as the distance to crop lines, as opposed to the vegetation index of neighbouring pixels and texture features.

Table 2: Selected features ordered by decreasing information gain for the sunflower and maize cases.

\begin{tabular}{cccc}
\hline \hline \multicolumn{2}{c}{ Sunflower } & \multicolumn{2}{c}{ Maize } \\
\hline Feature & Channel & Feature & Channel \\
\hline Mean & ExG & Mean & ExG \\
Mean & Green & Energy & ExG \\
Mean neighbours & ExG & Homogeneity & ExG \\
Skewness & ExG & Contrast & ExG \\
Mean & Blue & Mean neighbours & ExG \\
Skewness & Green & Mean & Green \\
Energy & Green & Mean & Blue \\
Homogeneity & ExG & Standard deviation & ExG \\
Contrast & ExG & Correlation & ExG \\
Energy & ExG & Contrast & Green \\
\hline
\end{tabular}

"neighbours" features refer to the second set of spatial features proposed in this paper.

\subsection{Feature selection in maize}

Finally, an experiment has been conducted considering the maize field. The results can be seen in Table 1. The results in this case are not as satisfactory as in the case of sunflower crop. However, the same conclusions can be drawn: the use of the base statistics perform well (better than the use of all sources of information) but the use of a feature selection method yields a generally better performance. The selected features are also presented in Table 2. The number of common selected features for both crops is 7 out of 10 . The common features 490 are: mean - ExG channel, energy - ExG channel, homogeneity - ExG channel, contrast - ExG channel, mean neighbouring pixels - ExG channel, mean - green channel and mean - blue channel. From these results, several conclusions can be drawn. Firstly, the use of textures is useful when computed over the ExG channel. Secondly, the computation of crop lines can be obviated when using 
(apart from the ExG channel that plays a vital role for the classification) are the ones selected. Finally, there are some statistics that can be discarded (e.g. the kurtosis).

\section{Discussion}

Figures 6 and 7 show the result of the SVM trained with the selected features for a selected part of the sunflower and maize experimental fields, respectively. For the case of sunflower, the results are promising and satisfactory, since the algorithm tends to detect weed pixels accurately (even when these are close to the crop rows) and no salt-and-pepper effect is created. For the maize case, it 505 also solves the salt-and-pepper effect. Nonetheless, the method slightly overestimates weed pixels and presents problems associated to the classification of shadows. However, both the classification and user labelling is more complicated in this crop, as it can be seen analysing the two experimental fields.

Concerning within crop-row detection, the graphical results shown in Figures 6 and 7 are satisfactory. Moreover, although the results in Table 1 do not explicitly include a classification rate for within crop-row patterns, a clear conclusion can be drawn using the $M S$ result: since $50 \%$ of weed test patterns were selected as patterns lying inside crop-rows, the result of 91.50 of $M S$ in sunflower means that, in the worst case, $91.50 \%$ of weed objects are classified, which means that most patterns within crop-row are properly classified.
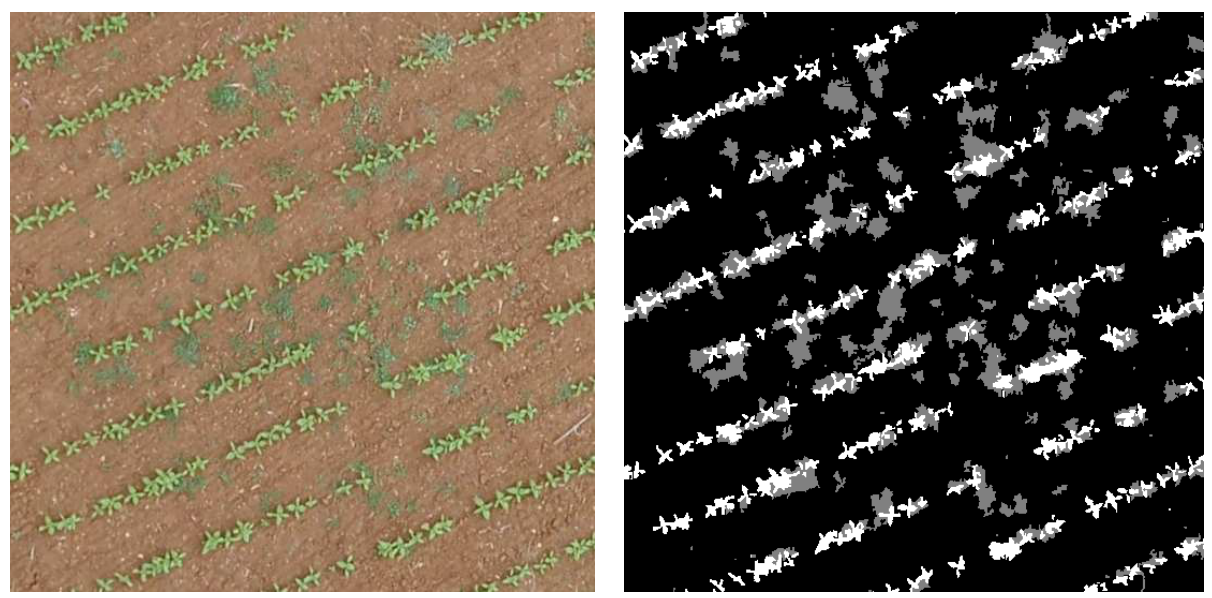

Figure 6: Representation of the results obtained for a selected part of the sunflower experimental field using the SVM method and the selected features. The plot on the left represents the original image and the one in the right the produced result, where each colour represents a class (black represents non-vegetation, white represents crop and grey corresponds to weeds).

It is worth mentioning that the only processing needed for the learning stage is the supervision of the images labelled by the designed method for pattern selection. Using only this information, the learning method leads to accurate 

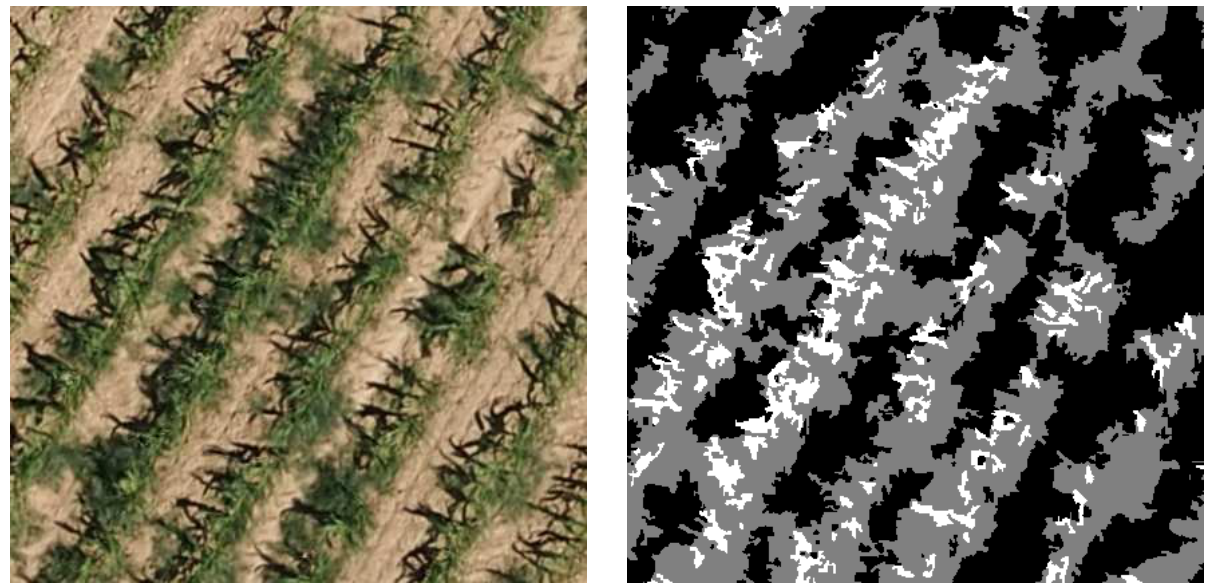

Figure 7: Representation of the results obtained for a selected part of the maize experimental field using the SVM method and the selected features. The plot on the left represents the original image and the one in the right the produced result (black represents non-vegetation, white represents crop and grey corresponds to weeds).

results. In light of the good results obtained, it can be stated that UAVs are 520 a suitable technique for the purpose of weed mapping, providing the perfect platform for flying a medium-size field with a reasonable spatial resolution and leaving open a wide line of future research.

With the output of the algorithm used, different treatments could be considered: a binary apply/not apply herbicide to the weed infested field section (if the of weeds are present, e.g. orass weeds) or the application of different herbicides (e.g. to control broadleaved, grass or resistant weeds). These treatment maps will be afterwards given to a specific software which will be part of a site-specific-treatment equipment.

Concerning the studied surface, although a plot of $100 \times 100$ might seem small, it is actually big in terms of data variability (due to the amount of pixels). Traditional platforms, such as satellites or planes, are able to capture plots of several kilometres, but they do not provide this spatial resolution and therefore the analysis provided in this paper is not feasible. In this sense, it is noteworthy that nowadays, the UAV technology is constantly evolving (Sun et al. 2011), and

535 these platforms might be able to fly bigger plots soon. Complemented with largescale machine learning methods (or big data approaches) these technologies might be a great tool for image analysis (specially in the agronomical scenario).

Several strengths and weaknesses of the methodology presented can be highlighted. Firstly, concerning the strengths, very few user intervention is needed, which is usually the main bottleneck of such a system. The features that have been selected are also easy to compute. Moreover, it can be seen that our procedure is not only capable of detecting weeds located out of crop rows, but also within-crop-rows themselves, which is one of the current challenges of the weed science literature. Finally, the OBIA approach helps dealing with the salt-and- 
${ }_{545}$ pepper effect common in pixel-based classification. Regarding the weaknesses of the proposal, one of them is that although most features helped in the classification of both crops, there are others that remain crop-dependent. Finally, another weakness is that the user intervention could be complicated when images are blurry or shadows can be found. it could be interesting to study the paradigm of incremental learning (Roscher et al. 2012). Under this setting, a trained model could be slightly modified in 
order to better fit new data, and this approach could ideally lead to good results

\section{Acknowledgements}

This work was financed by the Recupera 2020 Project (an agreement between CSIC and Spanish MINECO, EU-FEDER funds). Research of Mr. TorresSánchez and Dr. Peña was financed by the FPI and Ramón y Cajal Programs, respectively. Research of Dr. Gutiérrez and Dr. Hervás-Martínez has been subsidized by the TIN2014-54583-C2-1-R project of the Spanish Ministerial Commission of Science and Technology (MICYT), FEDER funds and the P11-TIC7508 project of the "Junta de Andalucía" (Spain).

\section{References}

Barla, A., Odone, F., \& Verri, A. (2003). Histogram intersection kernel for image classification. In Image Processing. Proceedings. 2003 International Conference on (pp. III-513-16 vol.2). volume 3.

Blaschke, T. (2010). Object based image analysis for remote sensing. ISPRS Journal of Photogrammetry and Remote Sensing, 65, 2-16.

Bunting, P., Clewley, D., Lucas, R. M., \& Gillingham, S. (2014). The remote sensing and gis software library (rsgislib). Computers and Geosciences, 62, $216-226$.

Burgos-Artizzu, X. P., Ribeiro, A., Guijarro, M., \& Pajares, G. (2011). Realtime image processing for crop/weed discrimination in maize fields. Computers and Electronics in Agriculture, 75, 337-346.

Carleer, A., \& Wolff, E. (2004). Exploitation of very high resolution satellite data for tree species identification. Photogrammetric Engineering 85 Remote Sensing, 70, 135-140.

Castillejo-González, I. L., Peña, J. M., Jurado-Expósito, M., Mesas-Carrascosa, F. J., \& López-Granados, F. (2014). Evaluation of pixel-and object-based approaches for mapping wild oat (avena sterilis) weed patches in wheat fields using QuickBird imagery for site-specific management. European Journal of Agronomy, 59, 57-66.

Clewley, D., Bunting, P., Shepherd, J., Gillingham, S., Flood, N., Dymond, J., 620 Lucas, R., Armston, J., \& Moghaddam, M. (2014). A python-based open source system for geographic object-based image analysis (geobia) utilizing raster attribute tables. Remote Sensing, 6, 6111.

Comba, L., Gay, P., Primicerio, J., \& Aimonino, D. R. (2015). Vineyard detection from unmanned aerial systems images. Computers and Electronics in Agriculture, $114,78-87$. 
Cortes, C., Mohri, M., \& Rostamizadeh, A. (2012). Algorithms for learning kernels based on centered alignment. Journal of Machine Learning Research, 13, 795-828.

European Crop Protection Association (ECPA) (2015). Url: http://www.ecpa.eu/information-page/industry-statistics-ecpa-total, in-

dustry statistics. URL: http://www.ecpa.eu/information-page/ industry-statistics-ecpa-total.

Ghamisi, P., Couceiro, M. S., Benediktsson, J. A., \& Ferreira, N. M. (2012). An efficient method for segmentation of images based on fractional calculus and natural selection. Expert Systems with Applications, 39,

12407 - 12417. URL: http://www.sciencedirect.com/science/article/

pii/S0957417412006756 doi:http://dx.doi.org/10.1016/j.eswa.2012. 04.078 .

Gutiérrez, P. A., López-Granados, F., Peña Barragán, J., Jurado-Expósito, M., $640 \quad \&$ Hervás-Martínez, C. (2008). Logistic regression product-unit neural networks for mapping Ridolfia segetum infestations in sunflower crop using multitemporal remote sensed data. Computers and Electronics in Agriculture, 64, 293-306.

Hall, M., Frank, E., Holmes, G., Pfahringer, B., Reutemann, P., \& Witten, I. H. (2009). The weka data mining software: An update. SIGKDD Explor. Newsl., $11,10-18$.

Herold, M., Liu, X., \& Clarke, K. C. (2003). Spatial metrics and image texture for mapping urban land use. Photogrammetric Engineering $\mathcal{E}$ Remote Sensing, 69, 991-1001.

Horizon 2020 (). Url: http://ec.europa.eu/programmes/horizon2020/. URL: http://ec.europa.eu/programmes/horizon2020/.

Hung, C., Xu, Z., \& Sukkarieh, S. (2014). Feature learning based approach for weed classification using high resolution aerial images from a digital camera

1 mounted on a uav. Remote Sensing, 6, 12037. URL: http://www.mdpi.com/ 2072-4292/6/12/12037, doi:10.3390/rs61212037.

Jain, A. K. (2010). Data clustering: 50 years beyond k-means. Pattern Recognition Letters, 31, 651-666.

Johnson, B., \& Xie, Z. (2011). Unsupervised image segmentation evaluation and refinement using a multi-scale approach. ISPRS Journal of Photogrammetry and Remote Sensing, 66, 473-483.

Lucieer, A., Turner, D., King, D. H., \& Robinson, S. A. (2014). Using an unmanned aerial vehicle (uav) to capture micro-topography of antarctic moss beds. International Journal of Applied Earth Observation and Geoinformation, 27, 53-62. 

manned aerial vehicle images. IEEE Transactions on Geoscience and Remote Sensing, 52, 1635-1647.

Mountrakis, G., Im, J., \& Ogole, C. (2011). Support vector machines in remote sensing: A review. ISPRS Journal of Photogrammetry and Remote Sensing, $66,247-259$.

Oerke, E.-C. (2006). Crop losses to pests. The Journal of Agricultural Science, $144,31-43$.

Otsu, N. (1979). A Threshold Selection Method from Gray-level Histograms. IEEE Transactions on Systems, Man and Cybernetics, 9, 62-66.

675 Peña, J. M., Torres-Sánchez, J., de Castro, A. I., Kelly, M., \& López-Granados, F. (2013). Weed mapping in early-season maize fields using object-based analysis of unmanned aerial vehicle (UAV) images. PLOS One, 8, e77151.

Pérez-Ortiz, M., Gutiérrez, P. A., Peña, J. M., Torres-Sánchez, J., HervásMartínez, C., \& López-Granados, F. (2015a). An experimental comparison for the identification of weeds in sunflower crops via unmanned aerial vehicles and object-based analysis. In I. Rojas, G. Joya, \& A. Catala (Eds.), Advances in Computational Intelligence (pp. 252-262). Springer International Publishing volume 9094 of Lecture Notes in Computer Science.

Pérez-Ortiz, M., Peña, J., Gutiérrez, P., Torres-Sánchez, J., Hervás-Martínez, C., \& López-Granados, F. (2015b). A semi-supervised system for weed mapping in sunflower crops using unmanned aerial vehicles and a crop row detection method. Applied Soft Computing, 37, 533 - 544. doi http: //dx.doi.org/10.1016/j.asoc.2015.08.027

Pérez-Ruiz, M., de Santos, P. G., Ribeiro, A., Fernandez-Quintanilla, C., Peruzzi, A., Vieri, M., Tomic, S., \& Agüera, J. (2015). Highlights and preliminary results for autonomous crop protection. Computers and Electronics in Agriculture, 110, 150 - 161.

Regulation (EC) 1107/2009 and Directive 2009128/EC (2009).

Roscher, R., Waske, B., \& Frstner, W. (2012). Incremental import vector machines for classifying hyperspectral data. IEEE Transactions on Geoscience and Remote Sensing, 50, 3463-3473.

Sun, T.-Y., Huo, C.-L., Tsai, S.-J., Yu, Y.-H., \& Liu, C.-C. (2011). Intelligent flight task algorithm for unmanned aerial vehicle. Expert Systems

with Applications, 38, 10036 - 10048. URL: http://www.sciencedirect.

700 com/science/article/pii/S0957417411002193 doi/http://dx.doi.org/ 10.1016/j.eswa.2011.02.013. 
Tellaeche, A., Pajares, G., Burgos-Artizzu, X. P., \& Ribeiro, A. (2011). A computer vision approach for weeds identification through support vector machines. Applied Soft Computing, 11, 908-915.

Torres-Sánchez, J., López-Granados, F., De Castro, A. I., \& Peña-Barragán, J. M. (2013). Configuration and specifications of an unmanned aerial vehicle (UAV) for early site specific weed management. PLOS one, 8, e58210.

Woebbecke, D., Meyer, G., Von Bargen, K., \& D.A., M. (1995). Color indices for weed identification under various soil, residue, and lighting conditions. Trans-

1 actions of the ASAE, 38, 259-269. URL: http://cat.inist.fr/?aModele= afficheN\&cpsidt $=3503524$.

Yu, Q., Gong, P., Clinton, N., Biging, G., Kelly, M., \& Schirokauer, D. (2006). Object-based detailed vegetation classification with airborne high spatial resolution remote sensing imagery. Photogrammetric Engineering 83 Remote Sensing, 72, 799-811. 